\title{
A new technique to teach basic concepts of refraction and reflection of light
}

Jung Hye Yoo, Bok Hee Cho, Dae-Kyu Kim, Seung-Han Park

Jung Hye Yoo, Bok Hee Cho, Dae-Kyu Kim, Seung-Han Park, "A new technique to teach basic concepts of refraction and reflection of light," Proc. SPIE 9666, 11th Education and Training in Optics and Photonics Conference, 966610 (5 June 2009); doi: 10.1117/12.2208085

SPIE Event: Eleventh International Topical Meeting on Education and Training in Optics and Photonics, 2009, St. Asaph, United Kingdom 


\title{
A new technique to teach basic concepts of refraction and reflection of light
}

\author{
Jung Hye Yoo and Bok Hee Cho \\ Department of Housing, Child, and Family, Kyunghee University, Seoul, Korea \\ Dae-Kyu Kim and Seung-Han Park \\ Department of Physics, Yonsei University, Seoul, Korea
}

\begin{abstract}
Nature of light is a facinating subject of education and training for children. However, it is not easy to demonstrate and explain fundamental properties of the light to the primary \& secondary school students. In this paper, we will present a new technique to teach concept of refraction, reflection, and total internal reflection of light in glasses by utilizing deviation angles of a prism for various surrounding index-matching oils.
\end{abstract}

Keyword list: reflection, refraction, total internal reflection, deviation angle, prism, index matching oil

\section{INTRODUCTION}

Glass, developed in Mesopotamia around B.C. 1700, provides us daily necessaries, arts, and the beauty. In addition, basic behavior of the light in glasses such as refraction and reflection allow us to observe a distant star or to detect the virus in micro scale. Therefore, it is quite important for childern to understand basic concept of refraction and reflection of the light to the primary \& secondary students since it helps them to understand nature of light as well as modern optical instruments.

There have been some difficulties to explain basic concept of reflection and refraction to the primary \& secondary school students. If students understand the concept of a trigonometry, it may be easy for us to give them a clear idea of the law of reflection and refraction. However, unfortunately, most of primary \& secondary school students are not exposed on these concepts. Even though various materials and experiments have been developed to help students to get basic behaviors of the light, therefore, most of them are not easy for the primary \& secondary school students to understand them easily and clearly. In particular, some of these materials even require calculating the trigonometric problem and/or following the complicated experimental processes [1,2].

In order to teach basic concept of reflection and refraction for primary \& secondary school students, it is necessary to introduce a relatively simple technique and to develop experimental set-up, which can provide intuitive pictures for the reflection and refraction of light $[3,4]$. In this paper, we will demonstrate a new technique to teach refraction, reflection, and total internal reflection of light by using a prism and index- 
matching oils simultaneously. In particular, we have utilized that the deviation-angle changes as the refractive index of the index-matching oil increases.

\section{UNDERSTANDING OF OPTICAL PROPERTIES}

When a light traveling through a transparent medium encounters a boundary leading into another transparent medium, a part of the light is reflected and the other part enters the second medium. Since the incident light and reflected light are symmetrical with respect to the line which is perpendicular to the boundary, in general, the angle of incidence equals the angle of reflection. On the other hand, the light that enters the second medium is bent (or refracted) at the boundary. The aspect of refraction depends on the differences in dielectric constants between air and acrylic, between glass and water, etc., and on the angle of incidence, as shown in fig. 1(a), (b) [3,4].

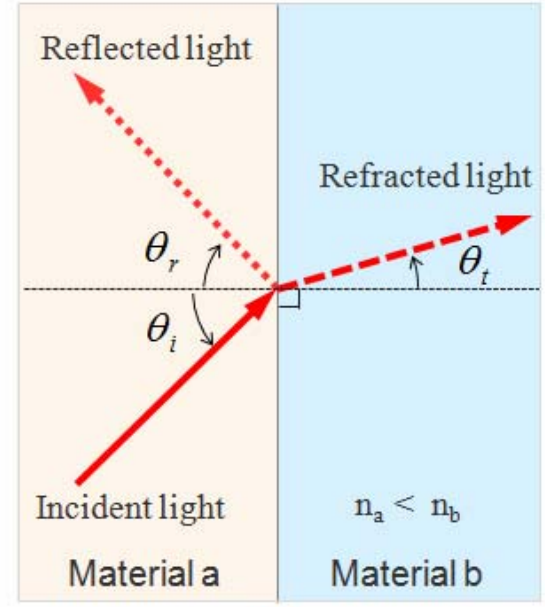

(a)

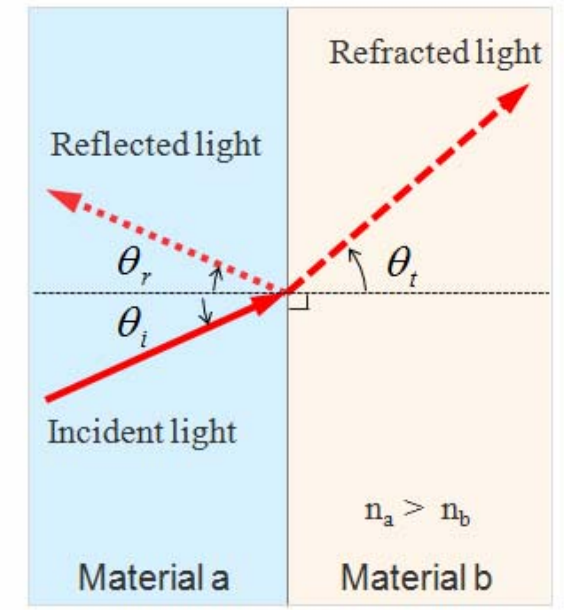

(b)

Fig. 1 Reflection and refraction of the light for (a) $n_{a}<n_{b}$ and (b) $n_{a}>n_{b}$

In order to instruct the concept of reflection and refraction to the students intuitively, it is useful to introduce the velocity of the light and its relation with refractive index. In fact, the optical properties of dielectric media are quite closely related to the refractive index. The velocity of the light in the medium is determined by refractive index by the equation (1) $[2,3,4]$.

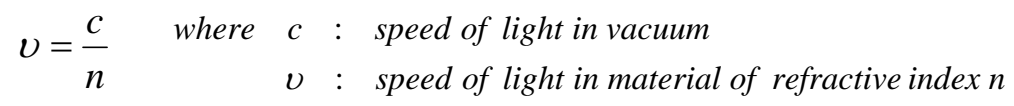

When the light travels in the air, its speed is approximately $3.00 \times 10^{8} \mathrm{~m} / \mathrm{s}$, but this speed is reduced down to approximately $2.00 \times 10^{8} \mathrm{~m} / \mathrm{s}$ when the light transmits inside a glass. The difference in the light speeds between two transparent media can be utilized to explain the refraction of the light at the interface between two media. A well-known example to explain the law of refraction for the primary \& secondary school 
students is as follows. What if students holding hands with each other jumped in at an angle from paved road to muddy land? As students hold hands tight with each other, their running speed is same and forms a straight line of students. When the right end of the running students reaches the muddy land as shown in Fig. 2., he slows down, while the left end remains on the paved road and moves at their original speed. This difference in speeds causes the line of students to pivot, and changes the direction of travel. Therefore, the relationship between the refractive index and the speed of light can be effectively utilized to provide an important physical property of the light to the students. Of course, we can explain also that the refraction angle increases as the difference of the refractive index in two media increases.
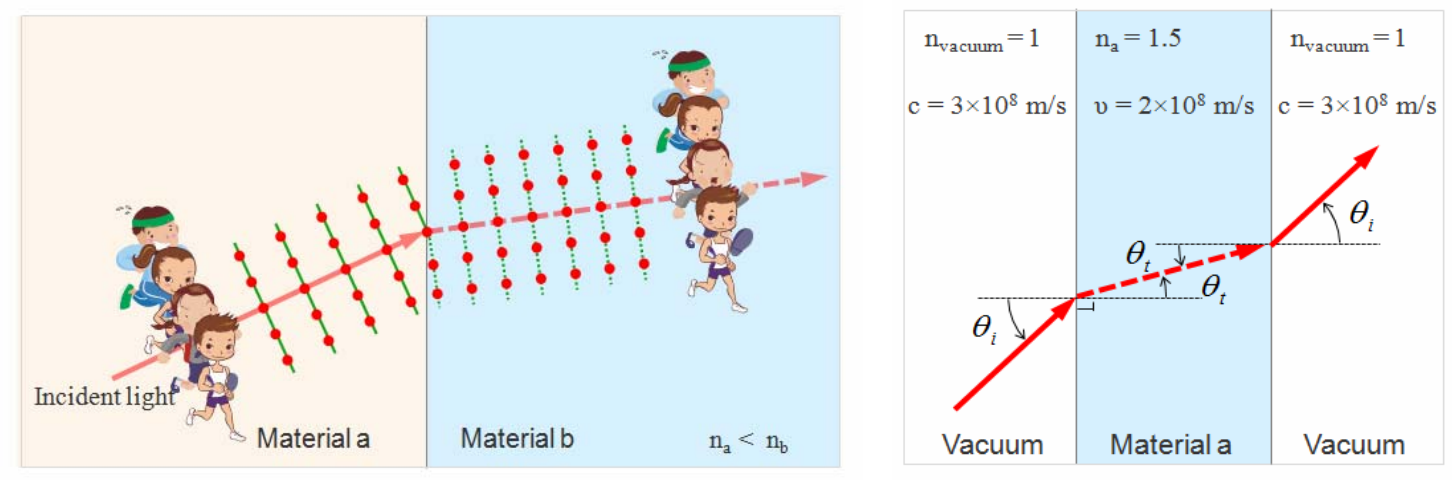

Fig. 2 Refraction of light

In addition, the behavior of light re-emerging into air, after passing through the substance, can be understood easily. When the light re-emerges into air, its speed instantaneously increases to its original value of $3.00 \times 10^{8} \mathrm{~m} / \mathrm{s}$. This phenomenon often makes the students quite confused since it is far different from what happens in daily life, for example, such as the reduced speed of a bullet firing through a block of wood. In this case, the speed of the bullet is reduced as it moves through the wood because part of its original energy is transferred to the wood in the form of heat due to the frictional force. When the bullet enters the air once again, therefore, it emerges at a speed just before leaving the block of wood. However, in case that the light travels in vacuum or the some transparent medium, it interacts with surrounding media by repeating absorption and radiation. In the glass, light travels from one glass atom to another at $3.00 \times 10^{8} \mathrm{~m} / \mathrm{s}$, the absorption and radiation that take place cause the average light speed through the material to fall to about $2.00 \times 10^{8} \mathrm{~m} / \mathrm{s}$. When the light re-emerges into the vacuum, the absorption and radiation of the light cease and the speed of the light returns to the original value. It is a similar situation that slow student in muddy land can run at their original speed in paved road.

An interesting effect called total internal reflection can occur when light is directed from a medium having a given index of refraction toward one having a lower index of refraction. Consider a light beam traveling in material $a$ and meeting the boundary between material $a$ and material $b$, where $n_{a}$ is greater than $n_{b}$. Various possible directions of the light are indicated as shown in Fig. 3(a). The refracted lights are bent away from the normal because $n_{a}$ is greater than $n_{b}$. At some particular angle of incidence $\theta_{c}$ called the critical angle, the refracted light moves parallel to the boundary. The critical angle to produce total internal reflection is small when $n_{a}$ is considerably greater than $n_{b}$. For example, the critical angle for a diamond in air which has refractive index of 2.4 is $24.5^{\circ}$. Any light inside the diamond that approaches the surface at an angle greater than this is completely reflected back into the crystal. This property, combined with proper faceting, causes 
diamonds to sparkle and colorful. The angles of the cutting facets are accurately adjusted so that light is "caught" inside the crystal through multiple internal reflections.

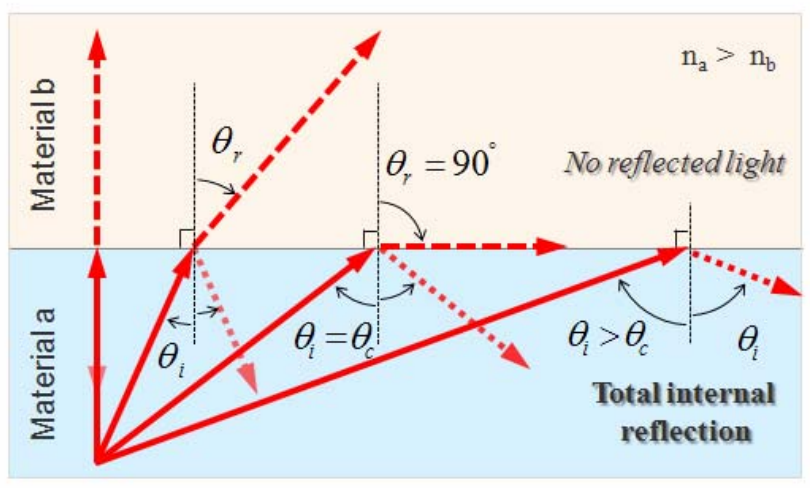

(a)

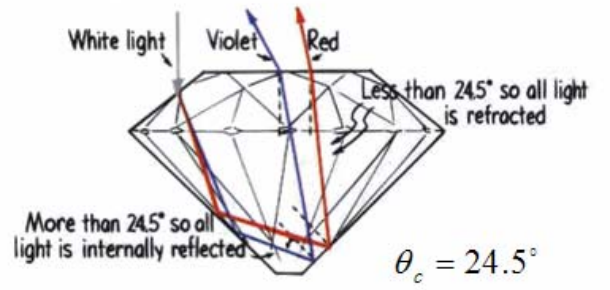

(b)

Fig. 3 Total internal reflection

\section{DESIGN OF EXPERIMENT}

Various experimental equipments for training basic concepts of reflection and refraction of the light have been developed. A typical experimental setup to provide better understanding of the law of reflection and refraction is shown in Fig. 4 [6]. In this experiment, the deviation angle of the refracted light by semicylinder block is measured. By tracing rays of light through a transparent semi-cylinder block and measuring the angles at the interfaces, the refractive index of material in the semi-cylinder vessel is calculated using Snell's law.
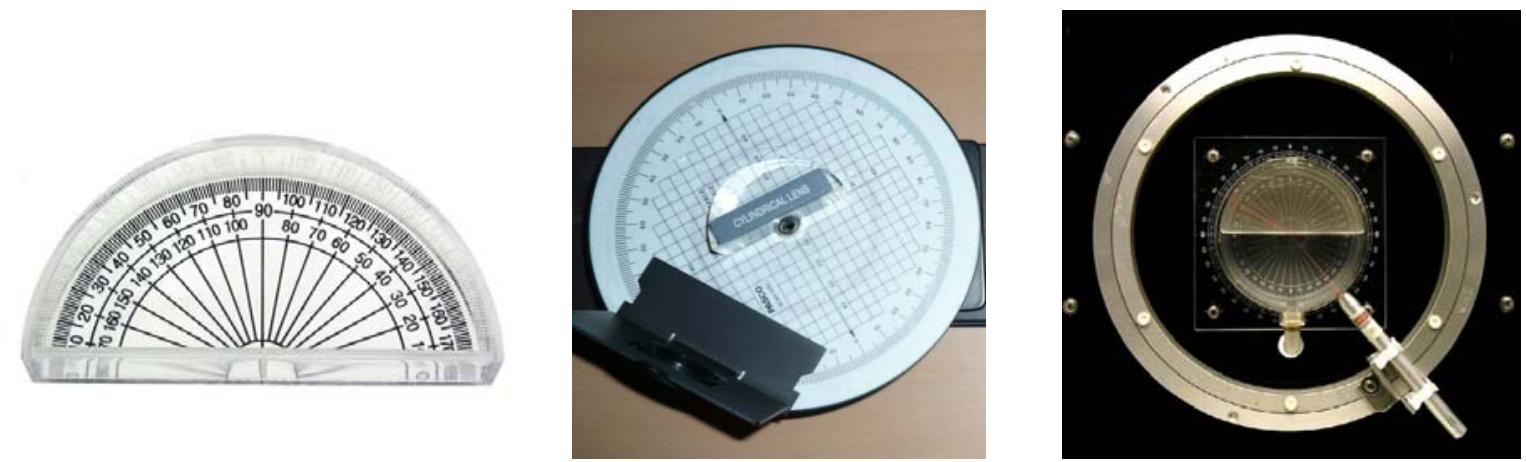

Fig. 4 Experiment for refraction and reflection of light

However such method may not be easy for the primary \& secondary school students to understand who have just encountered the phenomenon of refraction. They may feel frustrated by the associated mathematical problems, which act as unnecessary barriers at the level of their knowledge. Therefore, in this paper, we propose an effective experimental set-up in order to overcome these disadvantages. Our experimental set-up consists of glass prism, oils with various refractive indexes, and a water tank. 
Conventional glass prism is utilized and it has refractive index of 1.517. The prism is place into water tank, and index-matching oil is filled in water tank. When the light from the laser pointer is illuminated directly through the water tank and the prism, we can observe the two aspects of light propagation: reflection and refraction. The light is partly reflected in the interface separating two transparent materials, that is, oil and glass prism. Fig. 5(a) shows a schematic diagram of our experimental set-up.

In order to observe the light propagation more clearly, some of milk drop can be mixed with oil as a scattering sources. If the index-matching oil is not available, other substance, which can be obtained in our daily life, is very useful such as honey, water, and cooking oil. These substances can be filled up water tank in layers because of the difference of their densities. As the result of layered substances with different refractive index, various refracted lights can be observed in condition of fixed incident angle at the same time.

The incident light propagated through the prism from the left emerges refracted from its original direction of travel by an angle, called the deviation angle. The deviation-angle changes as the refractive index of the index-matching oil increases. With changing the incident angle, total internal reflection happens at the critical angle which varies according to the refractive index of the ambient medium. Fig. 3(b) shows a deviation angle of a prism for a given incident angle at $60^{\circ}$ as a function of refractive index of surrounding medium.
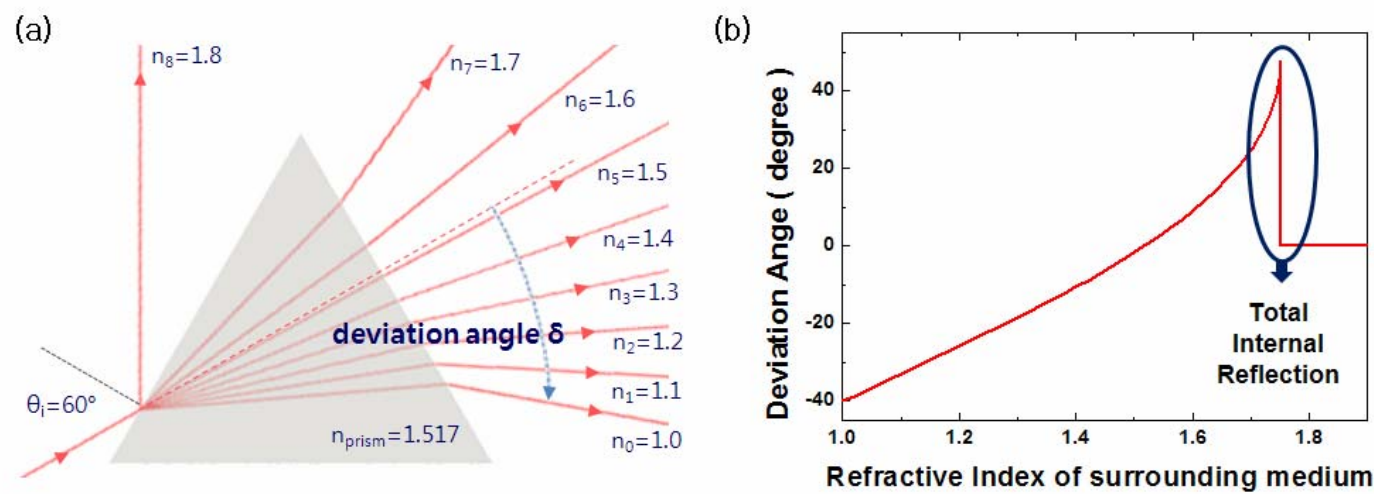

Fig. 5 (a) a schematic of our proposed experimental set-up (b) deviation angle as a function of refractive index of surrounding medium for the $60^{\circ}$ incident angle

This proposed experiment is intuitive for children who are unfamiliar with physical concepts such as reflection and refraction. Moreover, showing the process of emerging new scientific phenomena, total internal reflection, during experiment can make children feel attracted more to scientific experiments.

\section{CONCLUSION}

In this paper, we have demonstrated a simple technique to teach refraction, reflection, and total internal reflection of light by using a prism and index-matching oils simultaneously. This experimental mechanism helps us to observe the refraction of light between two media which have different refractive indices as well as the phenomena of total internal reflection. In particular, we have utilized that the deviation-angle changes as 
the refractive index of the index-matching oil increases. This experimental technique can be utilized as an effective teaching tool since it can offer students in various age group an integrated and entertaining way of learning basic concepts of light, such as refraction and reflection as well as total internal reflection simultaneously, by employing a simple and easily available equipment.

\section{References}

1. V. Cobb, and J. Cobb, Light Action! (SPIE press, Washington, 2005).

2. D. Halliday, R. Resnick, and Jearl Walker, Fundamentals of physics (John Wiley \& Sons, 2008).

3. E. Hecht, Optics (Addison Wesley, New York, 2002).

4. F. A. Jenkins, and H. E. White, Fundamentals of optics (McGraw-Hill, Tokyo, 1976).

5. S. L. Wong, and S. Mak, "Investigative studies of refractive indices of liquids and a demonstration of refraction by the use of a laser pointer and a lazy Susan," Physics Education, 43, 198 (2008). 